\title{
Interference during the implicit learning of two different motor sequences
}

\author{
Marianne A. Stephan - Beat Meier • Ariane Orosz • \\ Katja Cattapan-Ludewig • Alain Kaelin-Lang
}

Received: 4 July 2008 / Accepted: 6 May 2009 / Published online: 24 May 2009

(C) Springer-Verlag 2009

\begin{abstract}
It has been demonstrated that learning a second motor task after having learned a first task may interfere with the long-term consolidation of the first task. However, little is known about immediate changes in the representation of the motor memory in the early acquisition phase within the first minutes of the learning process. Therefore, we investigated such early interference effects with an implicit serial reaction time task in 55 healthy subjects. Each subject performed either a sequence learning task involving two different sequences, or a random control task. The results showed that learning the first sequence led to only a slight, short-lived interference effect in the early acquisition phase of the second sequence. Overall, learning of neither sequence was impaired. Furthermore, the two processes, sequence-unrelated task learning (i.e. general motor training) and the sequence learning itself did not appear to interfere with each other. In conclusion, although the long-term consolidation of a motor memory has been shown to be sensitive to other interfering memories, the present study suggests that the brain is initially able to
\end{abstract}

M. A. Stephan · A. Kaelin-Lang ( $\square)$

Department of Neurology, Movement Disorders Center,

Inselspital, Bern University Hospital, University of Bern,

3010 Bern, Switzerland

e-mail: alain.kaelin@dkf.unibe.ch

B. Meier

Department of Psychology, University of Bern, Bern, Switzerland

\section{A. Orosz}

Laboratory of Behavioural Neurobiology,

Swiss Federal Institute of Technology, ETH, Zurich, Switzerland

A. Orosz $\cdot$ K. Cattapan-Ludewig

Bern University Hospital of Psychiatry, Bern, Switzerland acquire more than one new motor sequence within a short space of time without significant interference.

Keywords Interference $\cdot$ Motor learning - Implicit sequence learning $\cdot$ Serial reaction time task $\cdot$ Age

\section{Introduction}

In daily life, humans often learn several motor patterns in succession. A dancer for example might first learn a specific series of steps and shortly afterwards another one. If such series of dancing steps are similar, the fact that a first series of steps have been learnt could facilitate the learning of a second series, but could also lead to confusion. Furthermore, the first series of steps may be forgotten by learning the second one. In other words, learning different motor patterns within a certain time-frame implies a potential for mutual influence.

Motor memory becomes less susceptible to such influences as time goes on, a process termed 'motor memory consolidation' (Krakauer and Shadmehr 2006). It has been suggested that during consolidation, the neural representations of motor memories change, such that they become functionally more stable (Shadmehr and Holcomb 1997). Accordingly, motor memories are thought to be unstable immediately after the acquisition and may therefore influence each other when two skills are learnt in direct succession (Robertson et al. 2004a, b). Many studies have investigated the long-term effect of such early mutual influences showing that when two skills are learnt in succession, the task that is learnt second causes reduced performanceimprovement or reduced retention in the task that is learnt first when the latter is retested hours to days after the original learning (Brashers-Krug et al. 1996; Shadmehr and 
Brashers-Krug 1997; Shadmehr and Holcomb 1997, 1999; Krakauer et al. 1999; Bock et al. 2001; Tong et al. 2002; Wigmore et al. 2002; Walker et al. 2003; Balas et al. 2007).

However, while much research has focused on such interference effects expressed after long time intervals, little is known about the immediate changes in the representation of a motor memory in the early acquisition phase of the learning process. Different memories might initially develop in parallel without mutual influence. Alternatively, a memory might instantaneously be overwritten by the acquisition of similar memories. In accordance with the latter suggestion, it has been shown that interference already leads to performance changes in the first minutes of motor memory formation. The learning of a visuomotor adaptation task disturbed the learning of a second task 15 min later (Miall et al. 2004) and, interference prevented individuals from adapting their reaching movements to one and then another new dynamic environment in immediate succession (Brashers-Krug et al. 1996; Shadmehr and Brashers-Krug 1997).

In contrast, it has been shown that two visuomotor sequences can be explicitly learned in immediate succession without interference and performance can be equally maintained when retested 5 min later (Walker et al. 2003). Furthermore, it is even possible to simultaneously learn two visuomotor sequences (Cock et al. 2002), as well as two sequences presented with different dimensional stimuli, such as visual and auditory sequences (Schmidtke and Heuer 1997), and temporal and spatial sequences (Shin and Ivry 2002).

Thus, the results concerning the occurrence and strength of early interference effects are contradictory and till now it is unclear under which conditions and at which time point in the learning process different motor memories interfere. It has been suggested that motor memories consist of multiple representations (e.g. movement goal or kinematics) that are processed in parallel at different rates (Verwey and Wright 2004). The sensitivity to interference might vary amongst these different representations. A motor memory might be more or less prone to interference based on the composition of its different underlying representations. This may explain, for example, differences between sequence learning, where subjects learn to chain single discrete movements into a cohering action and sensorimotor adaptation, where subjects adapt movements to environmental changes (Seidler 2006).

In the present study, we investigated whether interference effects occur in the early implicit sequence learning process with a variant of the well-known serial reaction time task (Nissen and Bullemer 1987; Willingham et al. 1989). In this task, subjects had to respond with the corresponding key presses to visual stimuli appearing, unbeknown to them, randomly or in a specific sequential order. As they learnt the task, the response time decreased due to at least two different learning processes: (1) sequence learning, that is, the learning of the specific sequential order of the visual stimuli, which does not occur when the stimuli are presented randomly. (2) Sequence-unrelated task learning, that is, the learning of motor task requirements as, for example, moving fingers appropriately to designated keys in response to visual stimuli.

Even though this sequence learning paradigm has been extensively used in the past, several facts remain unclear such as the role of sequence-unrelated task learning, age, and individual response velocity. In particular, findings regarding the effect of ageing on sequence learning are contradictory, with some studies reporting normal implicit sequence learning in older participants and others reporting deficits (Seidler 2006). Furthermore, we know from sports physiology that beginners have more scope for improvement than professionals with an already high performance level (Weineck 2002). Therefore, individuals with a slow response velocity at the beginning of the experiment would be expected to improve more than individuals with an initially fast response velocity, who might be closer to a ceiling effect. Finally, the task learning and sequence learning might be independent or related to each other. A participant who shows much improvement over time in the motor task learning aspect of the experiment might also be a good sequence learner or, in contrast to, might have little capacity to improve in sequence-specific learning.

The aims of the present study were therefore to determine (1) if there is an interference between motor memories in the early acquisition phase of two different motor sequences, (2) whether the two processes, sequence-unrelated task learning and sequence learning, are related to each other and, (3) whether there is an effect of age and individual response velocity on sequence learning and sequence-unrelated task learning.

\section{Methods}

Subjects

Fifty-five healthy subjects aged from 17 to 80 years without neurological or psychiatric disorders participated in the present study. Each subject was assigned to one of two different experimental conditions: sequence learning condition $(n=31$, mean age 47 , SD 19.31, range 17-75, 16 women, 29 subjects right handed, 2 left handed) and random control condition $(n=24$, mean age 46, SD 18.39, range $24-75,13$ women, 22 subjects right handed, 2 left handed). Handedness was assessed by the Edinburgh Handedness Inventory Score (Oldfield 1971). The study was approved by the local ethics committee and written informed consent was obtained from each subject. 
Paradigm

We used a variant of the classic serial reaction time task (Nissen and Bullemer 1987). Subjects had to respond with the corresponding key presses to flashing light stimuli appearing on a special Serial Response Box. Although the original version used one single sequence, we presented two different sequences repeatedly within separate learning blocks (sequence learning condition) and compared it with a random version of the task (random control condition), where the order of locations at which the lights appeared was random throughout the experiment. Sequence learning and sequence-unrelated task learning can both lead to a decrease in response time. When the visual stimuli appear randomly only task learning contributes to the decrease over time. In contrast, when the stimuli appear in a specific sequential order both sequence learning and task learning play a role. In order to extract the effect of sequence learning, classical paradigms take the response time difference in milliseconds (ms) between adjacent random and sequenced blocks as a measure of sequence learning. However with the classical analysis, the investigation of two sequences learned in alternate order would not be possible, because intermittent random blocks would impair the investigation of interference between sequences. To test our hypotheses, we introduced a second experimental group, which served as a random control condition. Because the two groups could have differed by chance in their general ability to perform the task, that is, in sequence-unrelated task learning, we introduced three random practice blocks at the beginning of both experimental conditions in order to test for such a possible confounding factor (see Fig. 2, R1-R3).

\section{Experimental procedure}

For stimulus presentation and response collection, we used the Serial Response Box (SRBox, model 200a, Psychology Software Tools Inc., Pittsburgh, PA, USA), a device with a row of four lights above four horizontally aligned keys, which was controlled by the E-Prime software (Psychology Software Tools Inc.).

Participants were instructed to press the corresponding key in response to the flashing light appearing just above the key and to do this as fast and as accurately as possible using index and middle fingers of their dominant hand (Fig. 1). The light turned off after a correct key press and the next light turned on $500 \mathrm{~ms}$ later. Participants were informed that they were going to perform a reaction time task and were not given any other information about the structure of the experiment. They were asked to concentrate on the task fully without talking or thinking about anything else.

In the sequence learning condition, the task consisted of eight blocks of 100 stimuli each. In blocks 1-3 (R1-R3) the

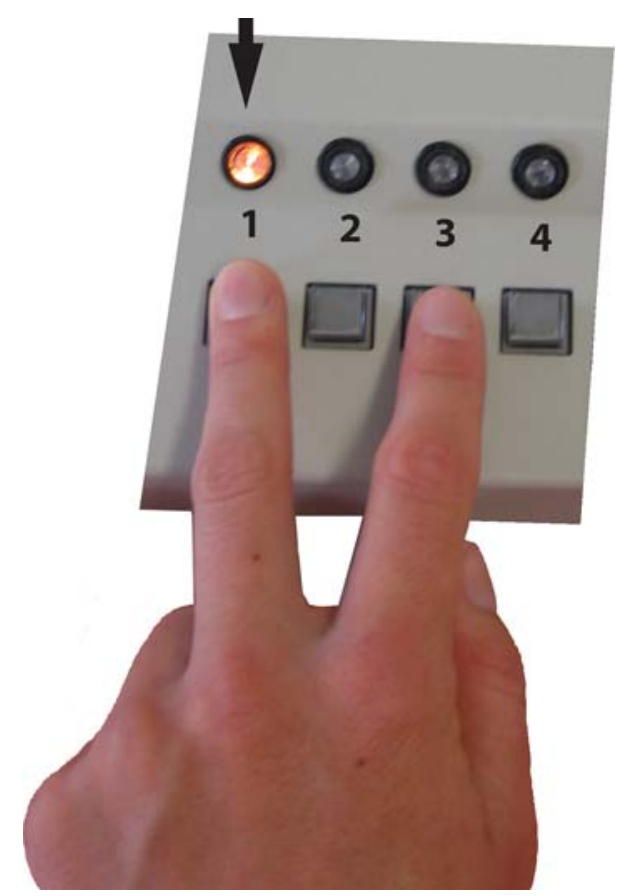

Fig. 1 Experimental setting for the left hand. Subjects had to press as fast as possible the corresponding key $(1-4)$ in response to the turning on of the lights (downward arrow), using their index finger for keys 3 and 4 and their middle finger for keys 1 and 2 (vice versa for right hand: index finger for keys 1 and 2, middle finger for keys 3 and 4)

stimuli appeared in random order. Afterwards, two different sequences were presented repeatedly within two separate 'learning-blocks' (A and $\mathrm{B}$ ) in 'learning-phase 1' and again in 'learning-phase 2' (Fig. 2). That is, block $4\left(\mathrm{~A}_{1}\right)$ consisted of ten repetitions of a specific 10-trial sequence ' $\mathrm{X}$ ' (keys 4-3-2-4-2-3-1-2-1-3), block $5\left(\mathrm{~B}_{1}\right)$ of ten repetitions of another sequence ' $y$ ' (keys 2-3-2-4-3-1-3-4-2-1), block 6 $\left(A_{2}\right)$ was a repetition of block 4 and block $7\left(B_{2}\right)$ a repetition of block 5 . In block 8 (R8), the stimuli again appeared in random order (Fig. 2). The duration of each block was about 2 min. Between 'learning-phase 1' and 'learningphase 2', a prolonged 5-min resting period was introduced in order to allow the beginning of motor memory formation processes. We assumed that memory formation processes require some time to occur (Robertson et al. 2004a, b). Thus, by the prolonged resting period, we aimed to increase the chance of detecting a possible interaction between the two developing motor memories. Furthermore, it allowed us to compare our results with those of other studies, in which also a 5-min interval between learning sessions was used (Goedert and Willingham 2002; Walker et al. 2003). The other blocks were separated by 1-min resting periods. In total, the whole experiment lasted for $30 \mathrm{~min}$. We kept the duration of sequence learning short (in total 20 repetitions per sequence, about $4 \mathrm{~min}$ ), in order to investigate early sequence acquisition. We assumed that at this time 


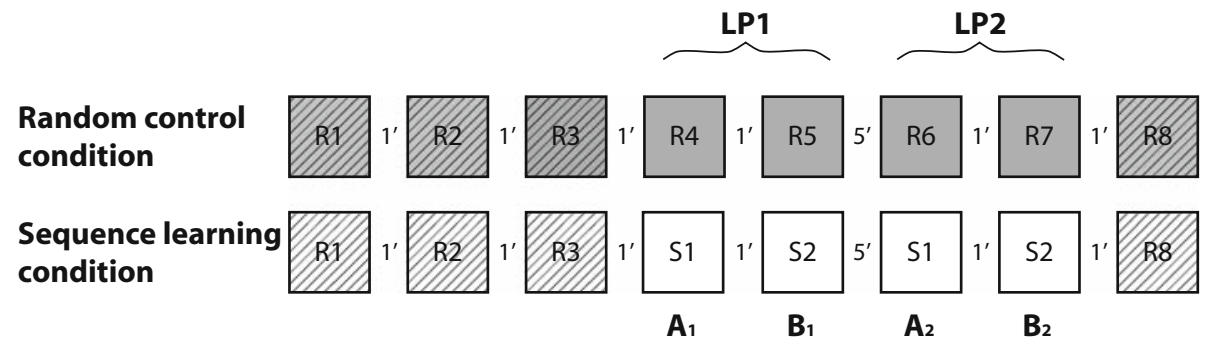

Fig. 2 Experimental procedure. Each subject was assigned to either the sequence learning condition (white) or the random control condition (grey). Stimuli were presented in eight blocks of 100 stimuli each. In blocks $R 1-R 3$, which were identical in both the experimental conditions, the stimuli appeared in random order. The 'learning-phase 1' ('LP1') enclosed two blocks $\left(A_{l}, B_{l}\right)$ in both conditions. Within the sequence learning condition, $A_{1}$ consisted of ten repetitions of a first

point, according to the traditional consolidation theory, motor memories should be most vulnerable to interference. The random control condition was analogous to the sequence learning condition, with the exception that the stimuli appeared randomly throughout the task.

Four keys enabled 16 different transitions between two keys (e.g. 1-1, 1-2, 1-3), whereas a 10-trial sequence consists of maximally ten different transitions, nine within the sequence itself and one between the sequences. To ensure the same degree of difficulty for the sequenced and the random blocks, they were all constructed from the same ten transitions. Each one of the ten transitions consequently occurred with a frequency of about $10 \%$ and the probability of a specific second key occurring after a specific first key remained constant for all blocks. In addition, an autocorrelation of the key transitions of the random blocks revealed a lack of periodicity for specific transitions. Furthermore, we presented the blocks with the sequences ' $x$ ' and ' $y$ ' counterbalanced and randomly in half of the subjects of the sequence learning condition in order $\mathrm{x}-\mathrm{y}-\mathrm{x}-\mathrm{y}$, in the other half of the subjects in order $\mathrm{y}-\mathrm{x}-\mathrm{y}-\mathrm{x}$. Following the preliminary data analysis, we decided to add additional trials to the last block R8. Therefore, in 15 out of 55 subjects, R8 consisted of only 20 instead of 100 trials. These subjects were excluded from data analyses involving R8. Four subjects of the sequence learning condition had the feeling that a specific sequence was repeated. However, only one subject had to be excluded due to a recall span of more than five keys indicating explicit knowledge (Robertson et al. 2004a, b).

\section{Data analysis}

Error rates were extremely low $(<2 \%)$ and were therefore not further analysed (Cohen and Robertson 2007). Only the response times of correct responses were included in the analysis. In each block, the first key press time was 10-trial sequence and $B_{1}$ of ten repetitions of a second different 10-trial sequence. Whereas, in the random control condition the stimuli appeared randomly in both $A_{I}$ and $B_{l}$. The 'learning-phase 2' ('LP2') was identical to ' $L P 1$ '. In block $R 8$, which was identical in both experimental conditions, the stimuli appeared in random order. The resting periods were $5 \mathrm{~min}$ between ' $L P 1$ ' and ' $L P 2$ ' and otherwise $1 \mathrm{~min}$. Hatched surfaces identical blocks for both conditions

discarded, because it was sometimes very prolonged due to a lack of attention when the task started. We calculated the medians of the response times per ten trials $(=1$ cycle), i.e. ten cycles per block for each subject, and used the means of these medians per 'block' as within-subject factor in all ANOVAs and ANCOVAs. Taking into account, the large inter-subject differences in individual response velocity, 'individual response times' were calculated for each subject (mean of the 10 cycle medians of R3) and used as a covariate in all ANCOVAs. We considered this 'individual response time' as a measure for the individual baseline performance before the beginning of the two 'learningphases'. $P$ values were always corrected for sphericity according to Huynh-Feldt and considered as significant if $<0.05$.

\section{Sequence learning}

Sequence learning was defined as the decrease in response time because the visual stimuli were presented in a specific sequential order. We performed a mixed-factorial ANCOVA with 'learning-phase' $(1,2)$ and 'learning-block' (A, B) as within- and 'condition' (random, sequence) as between-subject factor and, as mentioned above, with the 'individual response time' (mean of the 10 cycle medians of R3) as a covariate. We were interested in the main effect of 'condition' as a global indicator of sequence learning and also in the interaction 'learning-phase' $x$ 'condition', which would indicate a decrease in response time from 'learning-phase 1' to 'learning-phase 2' not only due to task learning, but also to sequence learning. In order to specifically investigate early sequence learning in block $A_{1}$, we performed a mixed-factorial ANOVA with 'block' $\left(\mathrm{R} 3, \mathrm{~A}_{1}\right)$ as within- and 'condition' (random, sequence) as betweensubject factor. An interaction 'block' $x$ 'condition', due to a stronger decrease in response time in the sequence condition, would indicate sequence learning in $\mathrm{A}_{1}$. In order to 
further test if learning of the first sequence did occur, we investigated whether the differences between R3 and $A_{1}$ were significantly different from zero with post hoc onesample $t$ tests for each condition. Similarly, as a specific analysis of sequence learning in 'learning-block B', we performed a mixed-factorial ANCOVA for the last two blocks with 'block' $\left(\mathrm{B}_{2}, \mathrm{R} 8\right)$ as within- and 'condition' (random, sequence) as between-subject factor and used one-sample $t$ tests for post hoc analyses.

\section{Interference}

Interference was defined as the difference in the extent of the response time decrease of ' $\mathrm{A}$ ' and ' $\mathrm{B}$ ' from 'learningphase 1 ' to 'learning-phase 2', reflecting an imbalance between the learning of ' $A$ ' and ' $B$ '. In order to investigate fine-grained and short lasting changes in response times within blocks, we included 'cycle' as additional within-subject factor in the same ANCOVA as for the investigation of sequence learning (see first paragraph Sect. "Sequence Learning"). We were interested in the interactions 'learning-phase' $\times$ 'learning-block' $\times$ 'condition' and 'learningphase' $\times$ 'learning-block' $\times$ 'condition' $\times$ 'cycle', since such interactions would reveal interference effects between the sequences.

\section{Task learning}

Task learning was defined as the decrease in response time due to the learning of sequence-unrelated mainly visuomotor task requirements (e.g. moving fingers appropriately in response to visual stimuli). To check for differences in task learning between the two experimental conditions in R1R3, we performed a mixed-factorial ANOVA with 'block' (R1-R3) as within- and 'condition' (random, sequence) as between-subject factor. Moreover, we reasoned that the two learning processes, task learning and sequence learning, might influence each other (sequence learning could either disturb or enhance simultaneous or ensuing task learning). Therefore, in order to check for differences between conditions in task learning following R3, we performed a mixedfactorial ANOVA with 'block' (R3, R8) as within- and 'condition' (random, sequence) as between-subject factor.

\section{Effects of age and 'individual response time' on interference, sequence learning and task learning}

For the purpose of using a Pearson correlation, we calculated the following parameters:

'Interference-parameter' $=$ (mean of cycle medians of ' $\mathrm{B}_{2}$ ' - mean of cycle medians of ' $\left.\mathrm{B}_{1}{ }^{\prime}\right)-($ mean of cycle medians of ' $A_{2}$ ' - mean of cycle medians of ' $A_{1}$ ').
'Sequence learning-parameter' = mean of cycle medians of $\mathrm{R} 8$ - mean of cycle medians of $\mathrm{B}_{2}$ (only calculated for those subjects in the sequence learning condition with complete R8, see Sect. "Experimental procedure").

'Task learning-parameter' = mean of cycle medians of $\mathrm{R} 1$ - mean of cycle medians of R8.

We correlated 'age' with the 'interference-parameter', the 'sequence learning-parameter', the 'task learningparameter' and the 'individual response time' (mean of R3). In addition, we correlated the 'individual response time' with the 'interference-parameter', the 'sequence learning-parameter', and the 'task learning-parameter' and finally, the 'task learning-parameter' with the 'sequence learning-parameter'.

\section{Results}

Sequence learning

The response times in both 'learning-phases' were significantly shorter in the sequence learning condition than in the random condition [Fig. 3, ANCOVA, 'condition', $F(1$, $52)=22.22, P<0.001$; mean difference between conditions: $\mathrm{A}_{1} 19 \mathrm{~ms}, \mathrm{~B}_{1} 17 \mathrm{~ms}, \mathrm{~A}_{2} 24 \mathrm{~ms}, \mathrm{~B}_{2} 20 \mathrm{~ms}$ ].

In both conditions, the response times were shorter in 'learning-phase 2' $\left(\mathrm{A}_{2}\right.$ and $\left.\mathrm{B}_{2}\right)$ than in 'learning-phase 1' $\left(\mathrm{A}_{1}\right.$ and $\left.\mathrm{B}_{1}\right)$ ['learning-phase', $F(1,52)=14.90, P<0.001$; 'learning-phase' $\times$ 'condition', $F(1,52)=0.53, P=0.47]$.

Response time changes from $\mathrm{R} 3$ to $\mathrm{A}_{1}$ were significantly different between the two conditions [ANOVA, 'block', $F(1,53)=18.80, P<0.001$; 'block' $\times$ 'condition', $F(1$, $53)=14.43, \quad P<0.001 ; \quad$ 'condition', $F(1,53)=0.12$, $P=0.74]$. In the random condition, there was a mean decrease in response time of $2 \mathrm{~ms}$ from $\mathrm{R} 3$ to $\mathrm{A}_{1}$, which was not significantly different from zero [one-sample $t(23)=0.38, P=0.71]$. In contrast, the decrease in response time of $24 \mathrm{~ms}$ in the sequence learning condition was significantly different from zero $[t(30)=5.91, P<0.001]$.

Also the response time changes from $\mathrm{B}_{2}$ to $\mathrm{R} 8$ differed significantly between conditions ['block' $\times$ 'condition', $F(1,37)=34.66, P<0.001$; 'condition', $F(1,37)=2.90$, $P=0.10]$, leading to a non-significant effect of 'block' [ANCOVA, 'block', $F(1,37)=0.23, P=0.64$ ]. In the random condition, the response time further decreased, on average by $7 \mathrm{~ms}$. However, this difference was not significantly different from zero, although there was a trend $[t(23)=2.00, P=0.06]$. In contrast, there was an increase in response time of $25 \mathrm{~ms}$ from the last sequenced to the final random block in the sequence learning condition, which differed significantly from zero $[t(15)=5.71$, $P<0.001]$. 


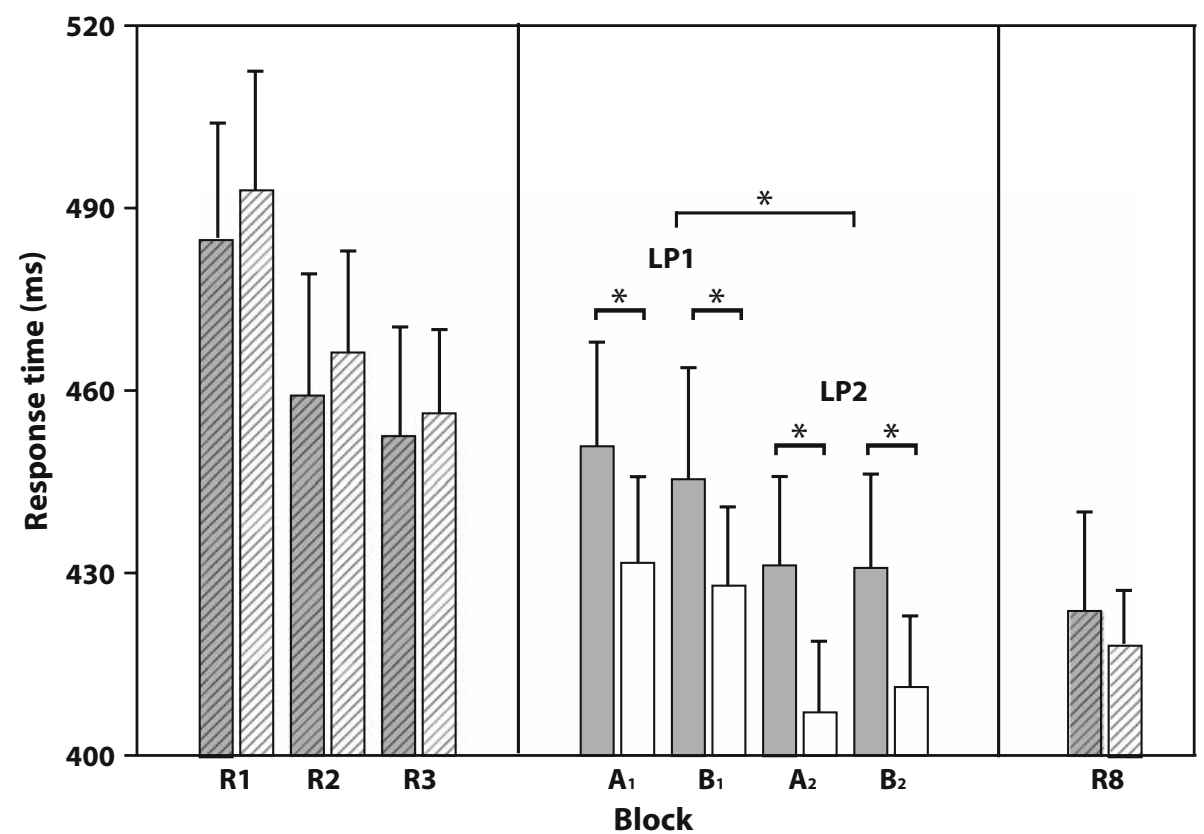

Fig. 3 Mean response times per block and experimental condition. There were no significant differences between the experimental conditions in blocks $R 1-R 3$ and $R 8$, in which the stimuli appeared randomly. In both the 'learning-phase 1' and 'learning-phase 2' (' $L P 1$ ' and ' $L P 2$ '), the response times in the sequence learning condition (white bars) were overall significantly shorter than those in the random

\section{Interference}

The decrease in response time from 'learning-phase 1 ' to 'learning-phase 2' was equally pronounced for both learningblocks ' $A$ ' and 'B' [ANCOVA, 'learning-phase' $\times$ 'learningblock', $F(1,52)=0.48, P=0.49$ ] and independent of the experimental condition ['learning-phase' $\times$ 'learningblock' $\times$ 'condition', $F(1,52)=0.21, P=0.65]$ (Fig. 3).

The response time changes across cycles within blocks differed between 'learning-phase 1' and 'learning-phase 2' depending on the 'learning-block' ['cycle' $\times$ 'learningphase' $\times$ 'learning-block', $F(8.05,418.52)=2.36, P<0.05]$ and also, on the condition ['cycle' $\times$ 'learningphase' $\times$ 'learning-block' $\times$ 'condition', $F(8.05,418.52)=$ 3.70, $P<0.001]$ : The response times were shorter in the random condition than in the sequence learning condition at the beginning of $\mathrm{B}_{1}$ (mean of random condition - mean of sequence condition in $\mathrm{B}_{1}$ : cycle $1-30 \mathrm{~ms}$, cycle 2 $-33 \mathrm{~ms}$ ), a phenomenon which did not occur in any other block.

\section{Task learning}

The overall response time in R1-R3 and the significant decrease in response time from R1-R3 did not differ between the two experimental conditions [Fig. 3, mean decrease from R1 to R3: $35 \mathrm{~ms}$, ANOVA, 'block', $F(1.59,84.02)=31.76$, control condition $(* P<0.001)$ ( grey bars). The decrease in response time from ' $L P 1$ ' to ' $L P 2$ ' $\left({ }^{*} P<0.001\right)$ was equally pronounced for both learning blocks ' $A$ ' and ' $B$ ' and independent of the experimental condition. Error bars standard error of the mean. Hatched surfaces identical blocks for both conditions. All subjects included (also those with incomplete $\mathrm{R} 8$, see text)

$P<0.001$; 'condition', $F(1,53)=0.06, P=0.80$; 'block' $\times$ 'condition', $F(1.59,84.02)=0.10, P=0.86]$.

The significant decrease in response time from R3 to R8 did not differ between the two experimental conditions either [Fig. 3, mean decrease from R3 to R8: $23 \mathrm{~ms}$, 'block', $F(1,53)=37.28, P<0.001$; 'condition', $F(1,53)=$ $0.002, P=0.97$; 'block' $\times$ 'condition', $F(1,53)=0.76$, $P=0.39]$.

Effects of age and 'individual response time' on interference, sequence learning and task learning

\section{'Age'}

'Age' did neither correlate with the 'interference-parameter' $(r=0.16, P=0.40)$, nor with the 'sequence learningparameter' $(r=0.06, P=0.83)$, but there was a moderate correlation with the 'task learning-parameter' $(r=0.31$, $P<0.05$ ). As expected, higher age also correlated with a longer 'individual response time' that is, older participants performed more slowly $(r=0.70, P<0.001)$.

\section{'Individual response time'}

The 'individual response time' did not correlate with the 'interference-parameter', although there was a trend ( $r=0.33, P=0.07)$, nor did it correlate with the 'sequence 
learning-parameter' ( $r=0.24, P=0.37)$. It did, however, correlate with the 'task learning-parameter' $(r=0.62$, $P<0.001)$ : Subjects with a longer 'individual response time' (mean of R3) tended to improve more from R1 to R8 than subjects with a shorter 'individual response time'.

Furthermore, the correlation between the 'task learningparameter' and the 'sequence learning-parameter' was not significant ( $r=-0.16, P=0.54)$.

\section{Discussion}

The main finding of this study is that two similar motor sequences can be implicitly learnt to the same degree, within the first minutes of the learning process, independent of whether they are introduced as a first or second sequence. This is shown by the absence of a difference between the learning of the first and the second sequence.

However, there is evidence for a slight, short-acting interference effect only with the first introduction of the second sequence after the initial learning of the first sequence: the response times at the beginning of the second learning-block were longer when the subjects had to respond to sequential stimuli than when they had to respond to stimuli in random order. Without the presence of repeating sequences, we would expect that the response times in the sequence condition would show the same pattern of change across cycles as in the random condition. Thus, if the pattern of change in the sequence condition differs from that in the random condition this has to be due to the presence of the repeating sequences. Therefore, we suggest that the learning of the second sequence was slightly and only briefly disturbed, due to the previous learning of the first sequence. It has been suggested that the interference in the early acquisition phase of motor learning is an expression of a disturbed working memory (Miall et al. 2004). Furthermore, it has been pointed out that working memory is involved in sequence learning (Pascual-Leone et al. 1993). The slight interference in the present study might thus have occurred because the memory of the first sequence competed with the memory of the second sequence for limited working memory space (Baddeley 1992). Such a competition might occur when persisting neural representations of an initial task (i.e. one that is presented and learnt first) disturb the processing of a subsequent task (i.e. one that is presented and learnt next) (Balas et al. 2007). However, we found only a minor interference effect which quickly disappeared and which, overall, did not lead to an impaired learning of either sequence. This is in line with other studies which measured interference effects in sequence learning after longer time intervals (Balas et al. 2007) but showed that sequences can initially be learned without interference in immediate succession (Walker et al. 2003) or even simultaneously (Schmidtke and Heuer 1997; Cock et al. 2002; Shin and Ivry 2002).

In contrast, another study revealed that learning one movement sequence disturbed the learning of another sequence already $5 \mathrm{~min}$ later (Goedert and Willingham 2002). However, as suggested previously (Krakauer and Shadmehr 2006), this might be a misleading interpretation. In the study by Goedert and Willingham, sequence learning was assessed in separate consecutive experimental sessions and defined as the difference between the mean response time of a final sequence block and the mean response time of two adjacent random blocks. There is evidence that, from one experimental session to the other, the response time decreased more due to sequence-unrelated task learning than to sequence learning (Goedert and Willingham 2002). This confounding factor could lead to a smaller difference between sequence- and adjacent random blocks in the second experimental session and therewith to an apparent impaired learning of the second sequence.

One could argue that we found only a slight interference effect because the extent of sequence learning was too small. However, three independent measures demonstrated sequence learning: first, response times decreased to a significantly greater extent in the sequence condition than in the random condition from the third random block R3 to the first sequence learning-block $A_{1}$. Second, response times in the four learning-blocks were significantly shorter when the stimuli were presented in sequential order than when they were presented randomly. Third, response times further decreased from the last learning-block $\mathrm{B}_{2}$ to the final random block $\mathrm{R} 8$ in the random condition, while they increased in the sequence condition, as a consequence of switching from a sequence to a random block. Thus, even though we cannot exclude that longer periods of sequence learning would have led to more interference, we think that the lack of relevant interference was not due to insufficient sequence learning.

Studies on adaptation learning found disturbed retention of a first learned task due to an interfering second task after longer time intervals of hours to days, but also immediate interference effects of a first on a second memory (BrashersKrug et al. 1996; Shadmehr and Brashers-Krug 1997; Shadmehr and Holcomb 1997, 1999; Krakauer et al. 1999; Bock et al. 2001; Tong et al. 2002; Wigmore et al. 2002; Miall et al. 2004). In contrast, we rather suggest that the brain is initially able to acquire at least two new motor sequences in succession within a short time interval without significant interference. Consistent with our work is also a study showing no significant decrease in sequence learning performance following the immediate application of transcranial magnetic stimulation (TMS) to the primary motor cortex (Robertson et al. 2005). Again, this suggests that the initial representation of motor sequence memories is less 
susceptible to interference than previously thought. Disturbing factors such as a competing memory or TMS might prevent the sequence memory trace from being strengthened without altering its initial expression (Robertson et al. 2005), which would lead to the detection of interference effects after longer time intervals but not in the early acquisition phase. Furthermore, the motor memory may not just consist of a single representation, but of multiple representations (Verwey and Wright 2004), each encoding a different functional attribute (Grafton et al. 1998). The relevance of these functional attributes might vary between different types of tasks. The representation of movement goals, for example, may be more important in sequence learning, whereas in adaptation learning the representation of movement kinematics may play a greater role. An interfering memory might disrupt only certain representations at a particular time but leave others unaffected. For example, it might predominantly disrupt the representation of the movements themselves leading to interference in adaptation tasks, whilst leaving the movement goals and therewith the sequence memory intact. Alternatively, in contrast to the traditional theories of learning which posit that new memories are labile and sensitive to interference from other memories before they are transferred to a long-term fixed and protected state, it has recently been proposed that memories can shift between active and inactive states (Nadel and Land 2000; Nader et al. 2000; Caithness et al. 2004). Thus, the shift into an inactive state might protect a sequence memory from being overwritten by another competing memory in the early acquisition phase.

To further explore the influence of several task inherent factors, the present study also aimed at clarifying the role of the individual response velocity in sequence learning and sequence-unrelated task learning. We found that subjects with a slow individual response velocity tended to show more sequence-unrelated task learning than fast subjects. However, the individual response velocity did not influence either sequence learning or the interference between the sequences.

Furthermore, since sequence-specific learning occurred mainly in the first learning-phase without substantial further improvement from the first to the second phase, we concluded that sequence learning is a rather fast process with most improvements occurring at the beginning. However, it is possible that sequence learning would have continued in a third learning-block. The finding that sequence learning is a quick process is in line with a previous study (Nissen and Bullemer 1987), in which a significant difference in the response times to random stimuli appeared after only seven repetitions of a specific sequence. In another study investigating the response times to randomly appearing stimuli, healthy subjects decreased their response time even across a much longer time interval of 24 blocks
(Laforce and Doyon 2001), instead of eight as in the current experiment.

As suggested previously (Cherry and Stadler 1995), sequence-unrelated task learning and sequence learning might influence each other when performed in succession. However, in our study, the decrease in response times due to task learning from the third to the last random block did not differ between the two experimental conditions, which indicates that sequence learning did not influence simultaneous or ensuing task learning. Also, there was no significant correlation between sequence learning and task learning. We thus suggest that these parameters indicate different learning processes, which do not influence each other.

Furthermore, our results support previous findings that old subjects respond more slowly than young subjects (Aizenstein et al. 2006; Seidler 2006). Nevertheless, their performance in sequence-unrelated task learning and in sequence learning was not impaired and old age was not related to an increased interference between the sequences. This is in line with other studies suggesting no difference between young and old adults in simple implicit sequence learning (Howard and Howard 1992; Dennis et al. 2006). Also, a study investigating visually guided sequential movements with a joystick revealed no sequence learning deficit in subjects of older age (Seidler 2006), and an fMRI study investigating concurrent implicit and explicit sequence learning found no difference in performance of implicit sequence learning between young and old subjects (Aizenstein et al. 2006). However, age-related deficits in sequence learning have been observed when sequence complexity was increased (Howard and Howard 1989; Curran 1997), and it is known that sensorimotor adaptation is more likely to be impaired in old subjects than sequence learning (Seidler 2006).

As mentioned above, slow subjects showed more sequence-unrelated task learning than fast subjects in our study. Because older subjects were slower, but age per se correlated only weakly with sequence-unrelated task learning, we suggest that the extent of sequence-unrelated task learning is mainly related to the individual response velocity, which is in turn influenced by other important factors besides age, for example, education or individual morphological characteristics. This suggestion is in line with another study in which "lower ability" older adults (determined by educational level, occupational status and verbal ability), who started with the longest response times at the beginning of the experiment, showed more sequence-unrelated task learning than "higher ability" older adults and younger adults (Cherry and Stadler 1995).

We conclude that (1) although the long-term neuronal processes responsible for the strengthening of a motor memory have been shown to be sensitive to other interfering memories and although short-term interference effects 
have been shown in adaptation learning, the brain is initially able to acquire at least two new motor sequences within a short space of time without significant interference. (2) Sequence-unrelated task learning and sequence learning are probably two dissociated processes, which do not influence each other. (3) Neither the individual response velocity, nor the age seems to have an effect on sequence learning or on the potential interference between sequences. However, a slower individual response velocity was related to a greater extent of sequence-unrelated task learning.

Acknowledgments The authors are grateful to Pietro Ballinari for statistical support, to Andros Tofield for text editing, and to Josephine Cock for critically reading and editing the manuscript. We also thank the anonymous reviewers for their detailed and constructive comments, which helped us greatly to improve the manuscript. This work was supported by a grant from 'Swiss Parkinson' to A.K.-L.

\section{References}

Aizenstein HJ, Butters MA, Clark KA, Figurski JL, Andrew Stenger V, Nebes RD, Reynolds CF, Carter CS (2006) Prefrontal and striatal activation in elderly subjects during concurrent implicit and explicit sequence learning. Neurobiol Aging 27:741-751

Baddeley A (1992) Working memory. Science 255:556-559

Balas M, Roitenberg N, Giladi N, Karni A (2007) When practice does not make perfect: well-practiced handwriting interferes with the consolidation phase gains in learning a movement sequence. Exp Brain Res 178:499-508

Bock O, Schneider S, Bloomberg J (2001) Conditions for interference versus facilitation during sequential sensorimotor adaptation. Exp Brain Res 138:359-365

Brashers-Krug T, Shadmehr R, Bizzi E (1996) Consolidation in human motor memory. Nature 382:252-255

Caithness G, Osu R, Bays P, Chase H, Klassen J, Kawato M, Wolpert DM, Flanagan JR (2004) Failure to consolidate the consolidation theory of learning for sensorimotor adaptation tasks. J Neurosci 24:8662-8671

Cherry KE, Stadler MA (1995) Implicit learning of a nonverbal sequence in younger and older adults. Psychol Aging 10:379-394

Cock JJ, Berry DC, Buchner A (2002) Negative priming and sequence learning. Eur J Cogn Psychol 14:27-48

Cohen DA, Robertson EM (2007) Motor sequence consolidation: constrained by critical time windows or competing components. Exp Brain Res 177:440-446

Curran T (1997) Effects of aging on implicit sequence learning: accounting for sequence structure and explicit knowledge. Psychol Res 60:24-41

Dennis NA, Howard JH, Howard DV (2006) Implicit sequence learning without motor sequencing in young and old adults. Exp Brain Res 175:153-164

Goedert KM, Willingham DB (2002) Patterns of interference in sequence learning and prism adaptation inconsistent with the consolidation hypothesis. Learn Mem 9:279-292

Grafton ST, Hazeltine E, Ivry RB (1998) Abstract and effector-specific representations of motor sequences identified with PET. J Neurosci 18:9420-9428

Howard DV, Howard JH (1989) Age differences in learning serial patterns: direct versus indirect measures. Psychol Aging 4:357-364
Howard DV, Howard JH (1992) Adult age differences in the rate of learning serial patterns: evidence from direct and indirect tests. Psychol Aging 7:232-241

Krakauer JW, Shadmehr R (2006) Consolidation of motor memory. Trends Neurosci 29:58-64

Krakauer JW, Ghilardi MF, Ghez C (1999) Independent learning of internal models for kinematic and dynamic control of reaching. Nat Neurosci 2:1026-1031

Laforce R Jr, Doyon J (2001) Distinct contribution of the striatum and cerebellum to motor learning. Brain Cogn 45:189-211

Miall RC, Jenkinson N, Kulkarni K (2004) Adaptation to rotated visual feedback: a re-examination of motor interference. Exp Brain Res 154:201-210

Nadel L, Land C (2000) Memory traces revisited. Nat Rev Neurosci 1:209-212

Nader K, Schafe GE, LeDoux JE (2000) The labile nature of consolidation theory. Nat Rev Neurosci 1:216-219

Nissen MJ, Bullemer P (1987) Attentional requirements of learningevidence from performance-measures. Cogn Psychol 19:1-32

Oldfield RC (1971) The assessment and analysis of handedness: the Edinburgh inventory. Neuropsychologia 9:97-113

Pascual-Leone A, Grafman J, Clark K, Stewart M, Massaquoi S, Lou JS, Hallett M (1993) Procedural learning in Parkinson's disease and cerebellar degeneration. Ann Neurol 34:594-602

Robertson EM, Pascual-Leone A, Miall RC (2004a) Current concepts in procedural consolidation. Nat Rev Neurosci 5:576-582

Robertson EM, Pascual-Leone A, Press DZ (2004b) Awareness modifies the skill-learning benefits of sleep. Curr Biol 14:208-212

Robertson EM, Press DZ, Pascual-Leone A (2005) Off-line learning and the primary motor cortex. J Neurosci 25:6372-6378

Schmidtke V, Heuer H (1997) Task integration as a factor in secondary task effects on sequence learning. Psychol Res Psychologische Forschung 60:53-71

Seidler RD (2006) Differential effects of age on sequence learning and sensorimotor adaptation. Brain Res Bull 70:337-346

Shadmehr R, Brashers-Krug T (1997) Functional stages in the formation of human long-term motor memory. J Neurosci 17:409-419

Shadmehr R, Holcomb HH (1997) Neural correlates of motor memory consolidation. Science 277:821-825

Shadmehr R, Holcomb HH (1999) Inhibitory control of competing motor memories. Exp Brain Res 126:235-251

Shin JC, Ivry RB (2002) Concurrent learning of temporal and spatial sequences. J Exp Psychol Learn Mem Cogn 28:445-457

Tong C, Wolpert DM, Flanagan JR (2002) Kinematics and dynamics are not represented independently in motor working memory: evidence from an interference study. J Neurosci 22:1108-1113

Verwey WB, Wright DL (2004) Effector-independent and effectordependent learning in the discrete sequence production task. Psychol Res 68:64-70

Walker MP, Brakefield T, Hobson JA, Stickgold R (2003) Dissociable stages of human memory consolidation and reconsolidation. Nature 425:616-620

Weineck J (2002) Optimales Training Leistungsphysiologische Trainingslehre unter besonderer Berücksichtigung des Kinder- und Jugendtrainings. Spitta, Balingen

Wigmore V, Tong C, Flanagan JR (2002) Visuomotor rotations of varying size and direction compete for a single internal model in motor working memory. J Exp Psychol Hum Percept Perform 28:447-457

Willingham DB, Nissen MJ, Bullemer P (1989) On the development of procedural knowledge. J Exp Psychol Learn Mem Cogn 15:10471060 